\title{
Affinity for, and localization of, PEG-functionalized silica nanoparticles to sites of damage in an ex vivo spinal cord injury model
}

\author{
Bojun Chen ${ }^{1}$, Mahvash Zuberi ${ }^{1}$, Richard Ben Borgens ${ }^{1,2^{*}}$ and Youngnam Cho ${ }^{1,3}$
}

\begin{abstract}
Background: Traumatic spinal cord injury (SCI) leads to serious neurological and functional deficits through a chain of pathophysiological events. At the molecular level, progressive damage is initially revealed by collapse of plasma membrane organization and integrity produced by breaches. Consequently, the loss of its role as a semi-permeable barrier that generally mediates the regulation and transport of ions and molecules eventually results in cell death. In previous studies, we have demonstrated the functional recovery of compromised plasma membranes can be induced by the application of the hydrophilic polymer polyethylene glycol (PEG) after both spinal and brain trauma in adult rats and guinea pigs. Additionally, efforts have been directed towards a nanoparticle-based PEG application. The in vivo and ex vivo applications of PEG-decorated silica nanoparticles following CNS injury were able to effectively and efficiently enhance resealing of damaged cell membranes.

Results: The possibility for selectivity of tetramethyl rhodamine-dextran (TMR) dye-doped, PEG-functionalized silica nanoparticles (TMR-PSiNPs) to damaged spinal cord was evaluated using an ex vivo model of guinea pig SCl. Crushed and nearby undamaged spinal cord tissues exhibited an obvious difference in both the imbibement and accumulation of the TMR-PSiNPs, revealing selective labeling of compression-injured tissues.
\end{abstract}

Conclusions: These data show that appropriately functionalized nanoparticles can be an efficient means to both 1.) carry drugs, and 2.) apply membrane repair agents where they are needed in focally damaged nervous tissue.

\section{Background}

Traumatic CNS injury causes sometimes fatal and often severe neurological and functional deficits. Breaches formed as a result of mechanical damage to the cell membrane readily triggers a series of adverse events compromising the plasma membrane's essential role, progressive disruption of nearby neuronal tissues, to eventual disruption-induced cell and tissue necrosis [1-5]. As promising strategies for circumventing or controlling such membrane disruption, innovative molecular - or cellular-based - therapeutic approaches are now emerging that shows the potential towards clarifying clinical applications [6-8]. Among them, considerable effort has been directed toward the discovery

\footnotetext{
* Correspondence: cpr@purdue.edu

'Center for Paralysis Research, Department of Basic Medical Sciences, School of Veterinary Medicine, Purdue University, West Lafayette, IN 47907, USA

${ }^{2}$ Weldon School of Biomedical Engineering, Purdue University, West Lafayette, IN 47907, USA

Full list of author information is available at the end of the article
}

and the administration of synthetic polymers, particularly polyethylene glycol (PEG) or poloxamer 188 (P188), with a hope to promote the enhancement of functional recovery and regeneration [9-17]. Such water-soluble synthetic polymers are capable of effectively restoring the barrier function of damaged membrane and experimental rescue of tissues and cells in a wide variety of model systems. We list a few of both seminal and contemporary examples of these wide ranging investigations: a) rescue of lethally shocked human fibroblasts in vitro using P 188 [17]; recovery from testicular reperfusion injury using the surfactant T-1100 [18]; functional and physiological recovery subsequent to IV injection of the tri-block polymer P188 in guinea pig models of SCI [19]; Restitution of permeabalized skeletal muscle [16]; interruption in the decline in force-induced deficits in contraction in a dystrophic mouse model using P 188 [20]; functional recovery and organelle reassembly in an SCI model using co-polymer - based micro particles [21].

\section{Biomed Central}


Furthermore, we have shown that parenteral PEG application (Topical, Intravenous, Intraperitoneal, and Subcutaneous) all significantly benefit physiological, anatomical, and functional outcome measures in adult guinea pig models of SCI [5,22], peripheral nerve injury [23], traumatic brain injury $[13,14,24]$, and in acute veterinary clinical cases of SCI in canines [15]. Others have experimented with the addition of Magnesium salts to the PEG solution in SCI (the magnesium having no effect on outcomes) [25] and found benefit in different types of adult rat SCI behavioral models [26,27].

In spite of these clear benefits, there are substantial obstacles for the development of further human clinical trials. Under certain dosage regimens, the molecular weight (MW) and aqueous concentration of PEG can be toxic [28-31]. Indeed, there is a concern for the safety issue of using low MW PEG, given that toxic complexes might be generated during the dissociation process of ethylene glycol into dimers and monomers [32,33]. Additionally, when given by intravenous routes, increased viscosity of PEG as a consequence of high concentration might cause problem in systemic circulation. From a practical standpoint, such limitations would not allow for sufficient flexibility in preparation and application of PEG, and might restrict the appropriate use of systemic/ intravenous polymers for maximum efficacy and efficiency. To overcome these problems, we have shifted our research focus toward the development of nanoparticle-based membrane applications systems. In previous studies, PEG-functionalized silica nanoparticles (PSiNPs) demonstrated a capability to seal damaged cells and tissues providing neuroprotection subsequent to spinal cord trauma [34-37]. Specifically, we reported the beneficial effects of PSiNPs after systemic application, including physiological recovery of somatosensory evoked potentials (SSEP) following severe compression of guinea pig spinal cords. PEG-tethered nanoparticles offer several advantages over systemic administration of solutions of these various polymers: i) the large surface areas of nanoparticles permit significantly greater PEG delivery at the particular cell/tissue regions of interest. While the systemic footprint of the polymer would likely be undetectable [37], ii) PEG-conjugated particles diminish the current challenges that could arise from the solution-based administration. Indeed, such nanocarrier systems not only minimize the amount of free drug in solution, but also prevent polymer degradation and inactivation upon administration, $[36,38]$ and iii) the small size of nanoparticles affords their internalization inside cells while evading uptake by phagocytic cells [39]. The latter is particularly important given that rescue of lethally poisoned PC 12 cells with Acrolein requires the internalization of PEG [40], also see Summary Figure 6, page 136 in [41]. Acrolein is a potent natural aldehyde

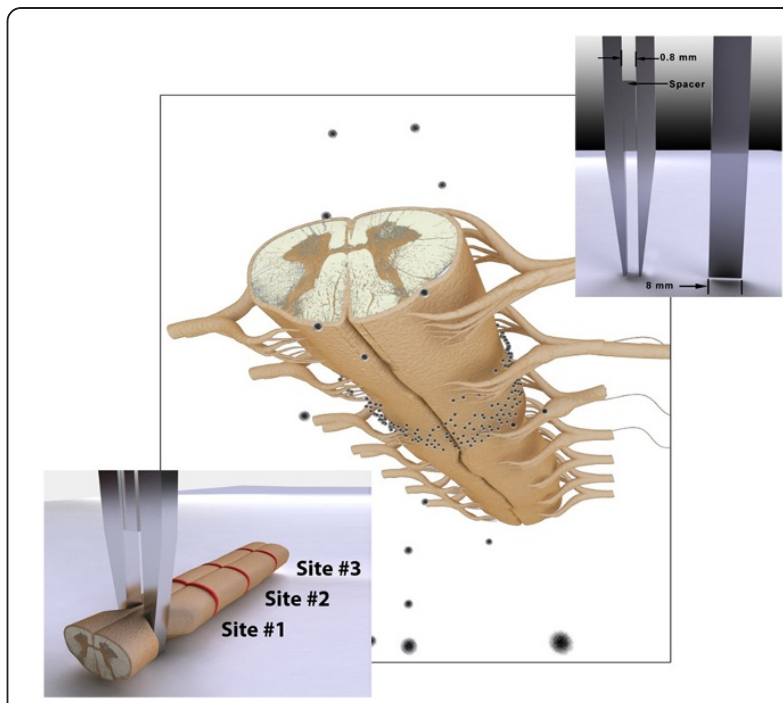

Figure 1 Schematic illustration of the compression injury to the ex vivo spinal cord tissue. The bottom left shows the compression injury and adjacent uninjured sites from with $1 \mathrm{~cm}, 2 \mathrm{~cm}$, and $3 \mathrm{~cm}$ gap (site \#1, \#2,\#3). The upper right shows the front and side view of modified forceps with a détente $0.8 \mathrm{~mm}$ in width when closed by pressure against the spacer. The isolated spinal cord was placed between the tips of the forceps to induce the compression injury by squeezing for $15 \mathrm{sec}$ against the spacer. The graphic is somewhat exaggerated for the purposes of illustration.

toxin created as an endpoint to LPO following mechanical trauma to cells [42].

The purpose of this study was to evaluate the selectivity of TMR-doped, PEG-functionalized silica nanoparticles (TMR-PSiNPs) during the repair of damaged spinal cord tissue.

Further understanding of the seminal events following injury-induced CNS cell and tissues damage relative to secondary injury mechanisms will likely provide appropriate and specific treatments for traumatic spinal cord and brain injury, and possibly other neurodegenerative disorders $[41,42]$.

\section{Results}

The suitable functionalization of the nanoparticles enables a possible preferential recognition of specific cells, facilitating binding and affinity-based endocytosis. Inspired by these important features, silica nanoparticles were functionalized with recognition elements, or PEG, on their surfaces, expecting localization toward particularly injured tissue (Figure 2).

As-synthesized, dye-doped silica nanoparticles with a mean diameter of $150 \mathrm{~nm}$ were used for all experiments detailed in this report. The preparation of dye-doped, PEG functionalized silica nanoparticles (TMR-PSiNPs) was initiated via the formation of silica nanoparticles with the entrapment of hydrophilic organic dye molecules (e.g., TMR-dextran) inside the silica pores during hydrolysis 

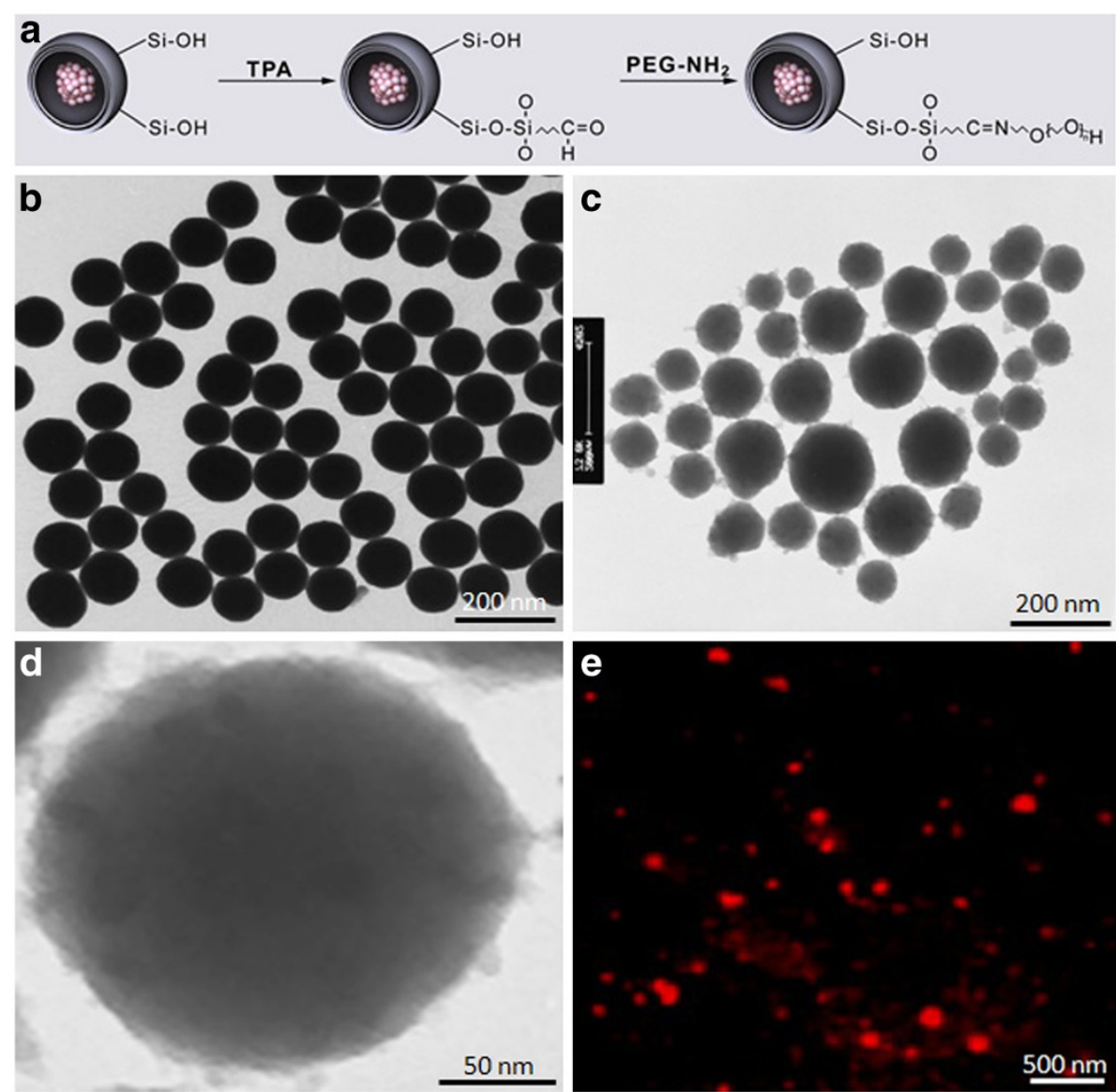

Figure 2 Decorated Peg-coated Nanoparticles. (a) Diagrammatic synthesis of TMR dye-doped, PEG-tagged silica nanoparticle (TMR-PSiNPs). The particles were separated from microemulsion by vortexing and centrifugating in acetone and ethanol, respectively. (b) (c) TEM images of bare silica nanoparticles and TMR-doped PEG-coordinated silica nanoparticles (TMR-PSiNPs) with a mean diasmeter of $150 \mathrm{~nm}$. (d) shows the magnified image of (c) to emphasize the presence of TMR dextran as a black dot in the silica structure. (e) Fluorescence microscopic image of TMR-PSiNPS.

and sol-gel polymerization of tetraethyl orthosilicate (TEOS) under the acidic condition (Figure 2(a)). Upon the preliminary particle preparation, surface modification was required to activate the surface with PEG layers by converting the end hydroxyl groups to aldehyde substituents through the reaction with TPA. Consequently, PEG- $\mathrm{NH}_{2}$ was conjugated to the aldehyde moiety through Schiff's base linkage (refer to Figure 2(a)). Figure 2(b) and (c) showed typical TEM images of bare silica nanoparticles and TMR-PSiNPs with mean diameters of $150 \mathrm{~nm}$, respectively. PEG-conjugated nanoparticles showed a flocculant surface morphology, indicating hydrophilic flexible chains of PEG were cross-linked on their surfaces. In addition, the higher magnification image of TMR-PSiNPs emphasizes the presence of TMR-dextran which appeared as black spots (Figure 2(d)). Figure 2(e) shows the fluorescence images of TMR-PSiNPs with optically manipulated fluorescent features, where silica network acts primarily as a supporting barrier to prevent the release of the dye
(TMR) from silica pore and to retain its luminophore activity. The chemical composition of modified silica surface was demonstrated by Fourier transform infrared (FT-IR) spectrometer and X-ray photoelectron spectroscopy (XPS), indicating the presence of PEG molecules on the silica surface.

Meanwhile, to evaluate the selective value of the PEGconjugated particles, TMR-PSiNPs were tested ex vivo with fifteen isolated spinal cord tissue samples and we assessed the degree of particle accumulation by measuring and quantifying the fluorescence intensity within tissue section as a function of distance from the injured area. Figure 3 displays total fluorescence intensity and distribution of TMR-PSiNPs, as fluorescent features of those particles enable visualization through deep-tissue penetration, particularly within damaged tissues. Interestingly, compression injury induces intense deposition, or localization, of TMR-PSiNPs in close association with lesions where intense labeling in both gray and white 

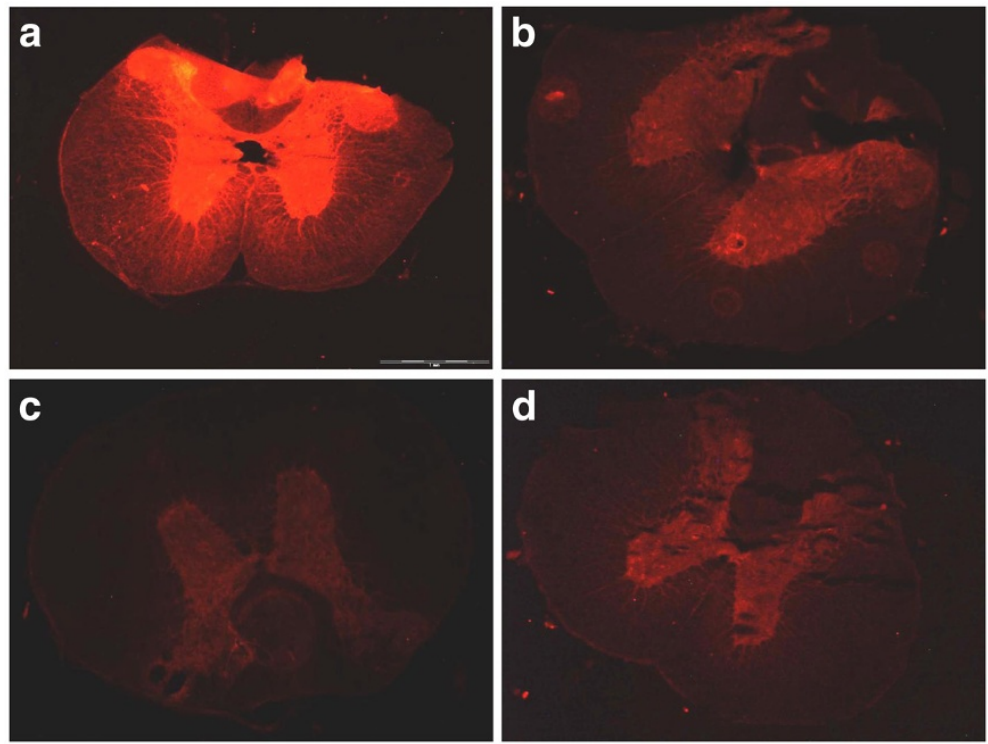

d

e

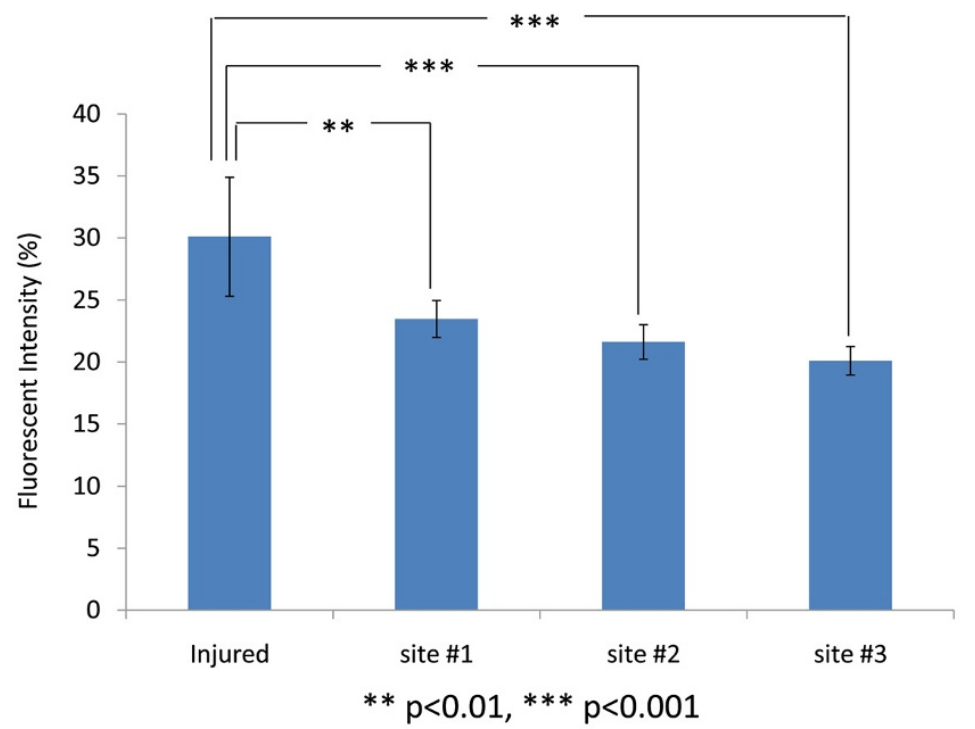

Figure 3 Localization of Fluorescently decorated Nanoparticles in Spinal Cord. (a) $\sim(\mathbf{d})$ The evaluation of injury-specific localization of TMRPSiNPs to injured and uninjured parenchyma using fluorescence microscopy. Crushed injured vs. uninjured spinal cord tissue are shown by fluorescence microscopy with $50 \mu \mathrm{m}$ thick frozen sections. (a) Compression injured cord section while (b) (d) uninjured spinal cord tissues with a distance of $1 \mathrm{~cm}, 2 \mathrm{~cm}$, and $3 \mathrm{~cm}$ from crush-injured spinal cord site. Obviously, crushed injury caused intense uptake of large amounts of TMRPSiNPs represented by significant labeling, especially in gray matter. (e) Quantification of fluorescent intensity in injured and uninjured spinal cord with background fluorescence normalized by subtraction of background fluorescence (see methods). Results are expressed as a percent of the uninjured values \pm SD $(n=5)$. ${ }^{* * P}<0.001,{ }^{* *} P<0.005$.

matter was observed as shown in Figure 3(a). In contrast to the highly fluorescent labeled injured tissue, tissues in close proximity from the injured area (e.g., site \#1, \#2, \#3) exhibited relatively modest staining, i.e. lower aggregation of particles (Figure 3(b d)). Figure 3(e) showed the quantification of the concentration of TMR-PSiNPs in injured and adjacent to injured (uninjured) tissue with modest distance from the focal injury.
Interestingly, the fluorescent intensity of uninjured tissue sections was about $40 \%$ lower than that in the injured tissue. As a control, bare TMR-doped silica nanoparticles (i.e. not coupled to PEG) were employed to observe such adsorption and accumulation within specific injury sites.

In Figure 4, a significant difference in fluorescence intensity between injured and normal tissues was not 


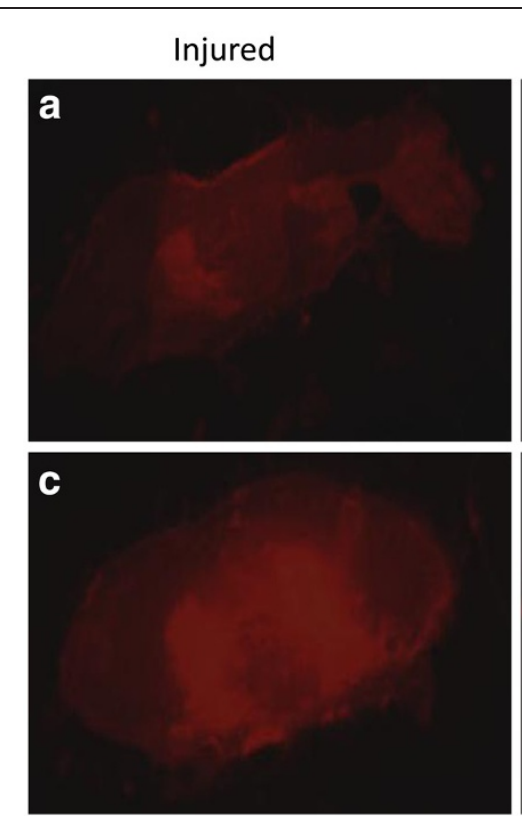

Site \#2
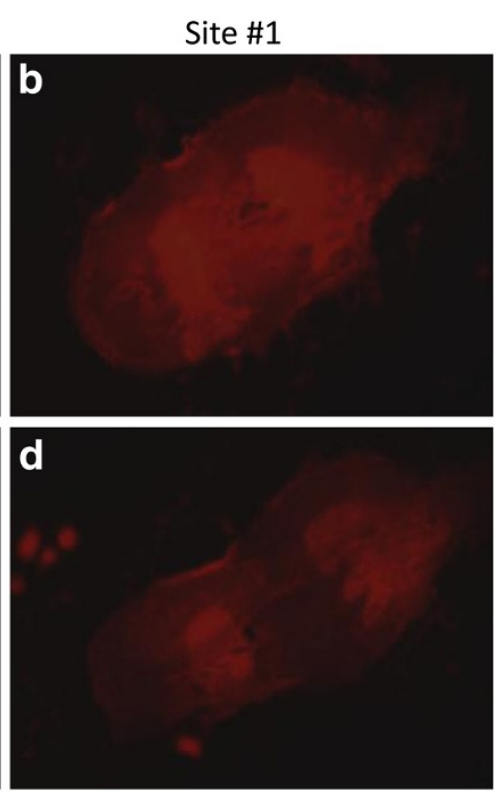

Site \#3

\section{e}

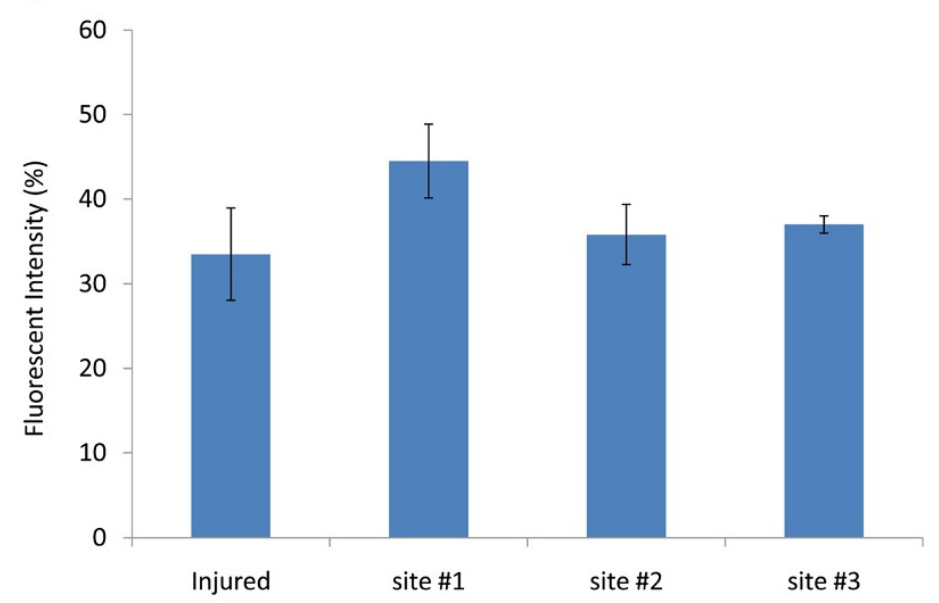

Figure 4 Nanoparticle Labeling in Injured and Uninjured Spinal Cord. (a) $\sim($ d) The evaluation of accumulation of TMR-SiNPs (without PEG conjugation) to injured and uninjured parenchyma using fluorescence microscopy. (a) Compression injured cord section, and (b) $\sim(\mathbf{d})$ uninjured spinal cord tissues with a distance of $1 \mathrm{~cm}, 2 \mathrm{~cm}$, and $3 \mathrm{~cm}$ from crush-injured spinal cord segment. It was not surprising that specific interaction in the uptake of TMR-SiNPs was not observed when PEG was not a component of the complex. (e) Quantification of fluorescent intensity in injured and uninjured spinal cord with background fluorescence normalized and with the subtraction of background fluorescence (see methods). Results are expressed as a percent of the uninjured values \pm SD $(n=5)$.

observed. Based on these observations, we suggest that PEG attached to particles is a key factor in promoting anatomical sealing of damaged neuron membranes supported by this affinity.. We also qualitatively studied the distribution of nanoparticles using SEM. Given the clear localization to the insult discussed above, an anatomical difference should be apparent. In support of these data, there appeared to be obvious differences in the localization of TMR-PSiNPs along the long axis of the cord with distance from the lesion. The higher- resolution SEM image revealed the localization of the particles within the lesioned tissue whereas the presence of particles was greatly decreased from the injured site as shown in Figure 5.

Indeed, the accumulation of nanoparticles to cord tissue was most reduced at site \#3, located a distance of $3 \mathrm{~cm}$ from the compression injury. Finally, the presence of silica particles within the tissues was also observed using the XPS spectrum (Figure 6). The high-resolution Si $2 p$ spectra exhibited an obvious signal through the 

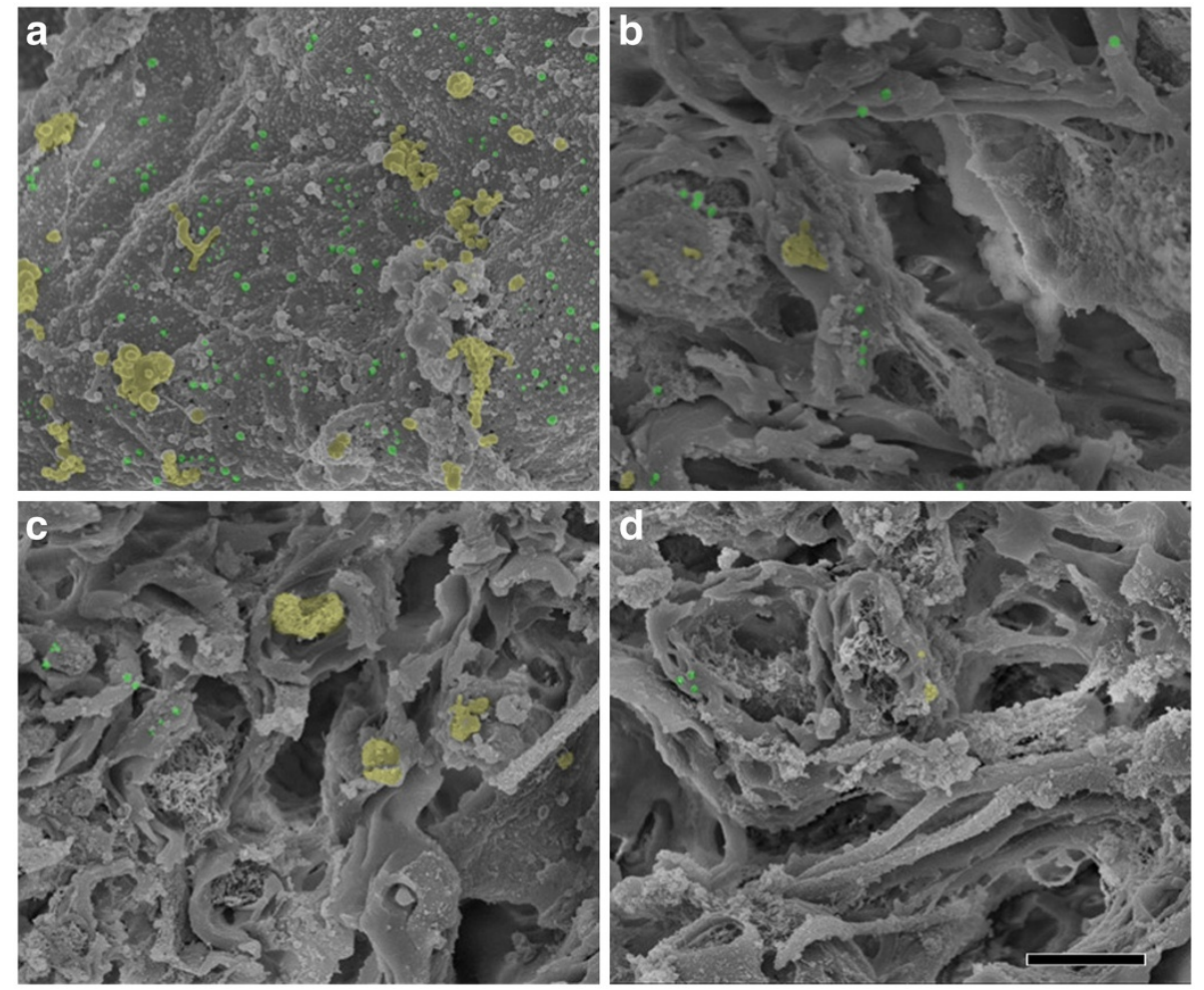

Figure 5 PSiNP Accumulations visualized by SEM. The cross sectional SEM images of injured spinal cord (a) and uninjured spinal cords located at site \#1 (b), site \#2 (c), and site \#3 (d) upon immediate post treatment with TMR-PSiNPs. Some, but not all, of individual and aggregated nanoparticles have been highlighted in green and yellow. Note the existence of dense particles in Figure 6a.

damaged tissue whereas $\mathrm{Si}$ peak was not observed through the intact cord tissues.

\section{Discussion}

Sealants, Fusogens, and the Damaged Cell Membrane

Since strongly hydrophilic polymers enabled the fusion of intact cells during the formation of hybridomas - it was a short jump in logic to hypothesized that parts of cleaved or diced cells might also be functionally reattached as well. This was first demonstrated by Bittner [43] who cut dissected giant axon from earthworm into two pieces and then subsequently functionally reattached them with PEG. The biophysics permitting the "fusogenic" properties of PEG and multi-block polymers (Poloxamers and Poloxamines) have been investigated for over three decades in cells and model membranes. As mentioned above, a complete understanding of the biophysics and mechanisms of fusion - let alone membrane repair - by these agents still alludes us. A discussion of the biophysics underlying fusion is beyond the scope of this investigative report - but we direct the interested reader to reviews of this subject $[4,41,42,44-46]$.

Once again we provide seminal thoughts from the clear leaders in this scholarship $[44,45,47]$ and contemporary understandings spanning decades to note the evolution in thinking about the mechanisms of action of polymer/surfactant based membrane fusion.

It is agreed that these hydrophilic polymers stabilize closely apposed, yet separate, cell membrane through interaction with, and alteration of, the aqueous phase of membrane amphilic phospholipids, as well as permit or induce the commingling of the hydrophic phase during membrane fusion. As Lee and others have taught membrane fusion is intimately associated with the organization of water at opposing membranes.

It is difficult to extrapolate experimental and theoretical understandings from polymer - based cell fusion studies to cell repair strategies. In the latter, it is likely that the significant hydrophilia of the repair agent is of prime importance. To rescue damaged but surviving cells, secondary dehydration of the "lesion" may be the most important outcome (refer to summary Figure 2 and 3; page 128 in [41]. In the absence of water within the breech, the lipid phases spontaneously reassemble ie., resolving together as an unbroken phase from the margins. This reestablishes the characteristic form of the membrane and the polar forces associated with the aqueous phase of the reconstituting membrane may reestablish much of the geometry of protein machinery of the membrane held in place in part by these polar 


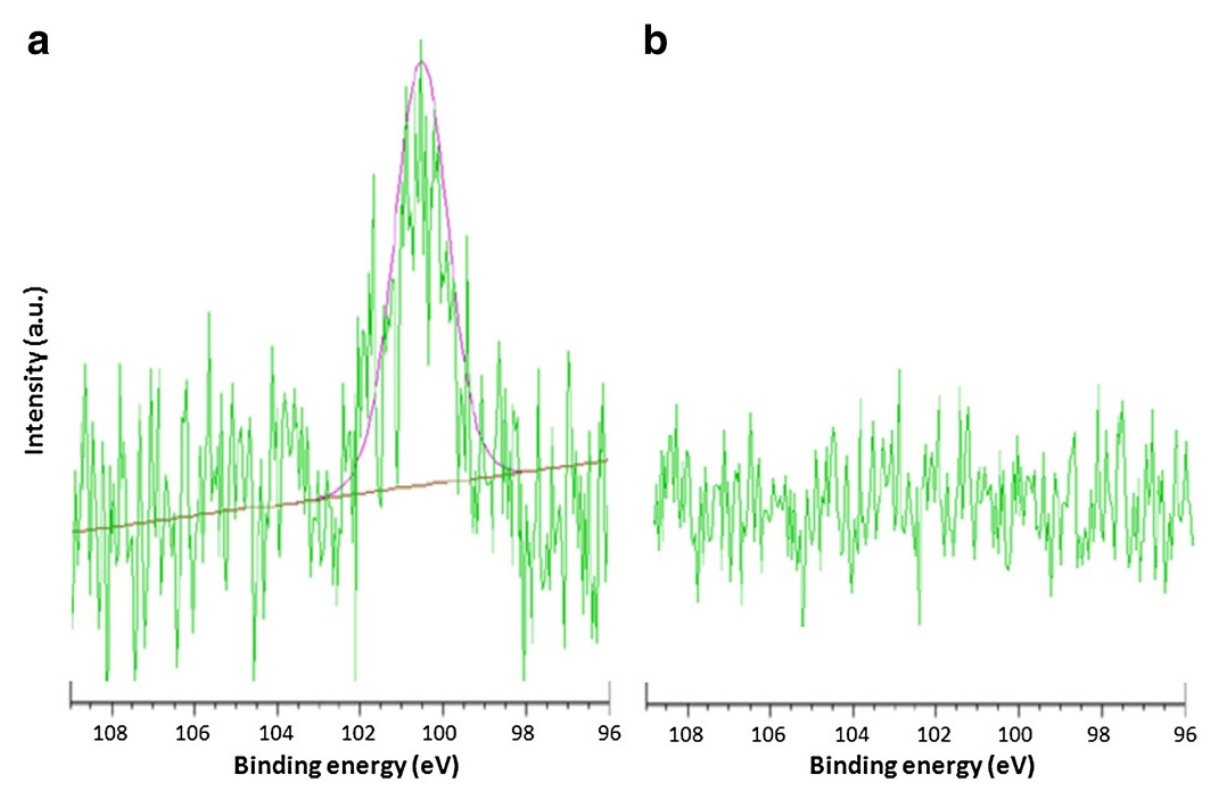

Figure 6 Evaluating the presence of TMR-PSiNPs in the injured vs. uninjured spinal cords by XPS. High-resolution XPS surface deconvolution analysis of Si $2 p$ was used to verify the existence of silica nanoparticles on (a) crush-injured cord tissue and (b) uninjured cord located at site \#2. Note that the compression injury increased the level of silica, identifying a precise targeting toward injured regions.

forces. These mechanisms, actual and theoretical, probe the ability of hydrophilic polymers to functionally reconstitute a variety of cell types and tissues and require more investigation.

\section{Evaluation of the localization of TMR-PSiNPs in injured and uninjured spinal cord tissues}

Recently, we have reported the ability of PEG-coated silica nanoparticles (PSiNPs) in the restoration of integrity of the structurally and physiologically damaged spinal cord tissues [34,37]. However, "targeting" (see below) the molecule to the specific cells/tissues becomes crucial to prevent adverse effects, and to enhance the density of PEG at only sites of damage. This then reduces the actual amount of PEG in systemic circulation at times after administration to vanishingly low.

In this study, crushed cords were incubated in the TMR-PSiNP solution for $15 \mathrm{~min}$ followed by the immersion in oxygenated Krebs' for an additional $30 \mathrm{~min}$. This process might readily account for the reduction in non-specific binding affinity between cord tissues and TMR-PSiNPs, thus indicating low background staining. Overall, our experimental results indicated that total accumulation as an indicative for the presence of TMR-PSiNPs subtly lowered in proportion to the distance from the injured area.

This study model - using the same cord as its own control has been verified and standardized. Briefly, the most sensitive indicator of membrane/tissue damage is an ionic current that flows into the compromised region
[48-50]. The magnitude of the current is indicative somewhat of the size of the injury. This is because all cells are inwardly negative and this membrane "battery" drives net current into an insult (be it a region of lowered membrane resistivity or an actual breach). We have measured such currents non- invasively entering axons and tracts of mammalian white matter with vibrating and neutating microprobe systems for the measurement of extracellular current [50]. In these ex vivo mammalian spinal cord segments - injury current is largely restricted to the region of a standardized compression (see below) and falls off steeply by 50 microns from it; see pages 5-6 in Zuberi et al. [50]. The comparative segments of cord are indeed adjacent to the injury zone and provide undamaged white matter for comparison to the central injury. This "control region" of this same cord shows no signs of anatomical or physiological damage. This model we believe is superior to using separate control cords.

We believe that the PEG-conjugated particles specifically provide selective binding to the damaged cell's surfaces, and thus allows for subsequent intracellular accumulation of significant amounts of nanoparticles with suitably adjusted affinities. In the uninjured segments, there appeared an obvious difference in the labeling of gray and white matter. This is likely due to the fact that neurons large clusters are only found in gray matter. Thus, gray matter may be a more useful site of absorption of particles compared to white matter composed of only nerve axons. This would be an especially 
important consideration in traumatic brain injury which involves loss of cerebral neurons.

Our data both extends and supports the induction of spontaneous reassembly of damaged membranes of nerves and other cells, their anatomical sealing, and the immediate recovery of nerve conduction through spinal cord by specific localization of PEG where it is most useful.

\section{New directions}

This paper is a part of a new investigation of several replacement strategies to the application of native PEG for the reasons given above. These also include polysaccharides that are also strongly hydrophilic, can act as "sealants", are biodegradable, and completely nontoxic $[41,51]$. Sugars derivatives such as Chitosan may also directly target membrane defects using a more appropriated nomenclature.

We [41] as others have used "targeting" to describe a synthetic polymer's mode of action in repairing cell defects. In reflection this is likely an illegitimate descriptor. It implies a more precise understanding of the mechanisms of specific recognition phenomenon such as observed in ligand / receptor coupling or immunological recognition strategies used in biology and medicine. Thus we have moved away from this term here to describe instead the affinity of symmetric polymers for regions of cell and tissue defects that have been empirically reported in SCI and TBI [14]. On the other hand - it may be completely accurate to describe Chitosan's ability to "recognize" and selectively adhere to cell membrane defects Here the molecular interactions of complex and disorganized lipids and reactive sites on chitosan are understood $[41,52,53]$.

\section{Conclusions}

In this study, we have observed accumulations of TMRPSiNPs in injured spinal cord tissue compared to nearby "undamaged" tissue. The results revealed a significant interaction of PEG-conjugated nanoparticles toward a compression injury, while there did not appear any apparent association to nearby uninjured tissues. An important fact explaining any aggregation at all in "uninjured tissues" is to recall the amount of handling and surgery used to provide the ex vivo samples of spinal cord for study. Indeed, this preferential targeting study strongly supports the initial observations with regard to PEG-mediated spontaneous reorganization, anatomical sealing, and recovery of conduction through guinea pig spinal cord white matter. These data also support PEG induced dramatic restoration of cerebral cells in damaged brain and recovery of function after such significant injury $[24,54,55]$.

\section{Methods}

Synthesis and functionalization of TMR-doped,

\section{PEG-functionalized Silica}

\section{Nanoparticles (TMR-PSiNPs)}

All chemicals were purchased from Sigma-Aldrich unless otherwise specified. TMR-doped, PEG-modified silica nanoparticles were synthesized according to the previously described procedure [37]. In brief, the mixture composed of $1.77 \mathrm{~mL}$ of Triton X-100, $1.8 \mathrm{~mL}$ of $\mathrm{n}$-hexanol, $7.5 \mathrm{~mL}$ of cyclohexane, and $0.5 \mathrm{~mL}$ of $1 \%$ aqueous tetramethyl rhodamine-dextran (TMR-dextran, Invitrogen) solution was prepared to form water-in-oil (W/O) reverse microemulsion by adjusting solution $\mathrm{pH}$ to 2.0 . Then, 100 $\mu \mathrm{L}$ of tetraethyl orthosilicate (TEOS) was slowly added to the mixture with vigorous stirring. The solutions were stirred for another $1 \mathrm{hr}$ until $60 \mu \mathrm{L}$ of $\mathrm{NH}_{4} \mathrm{OH}$ was added to the solution to terminate polymerization reaction. By adding acetone, the fluffy white precipitate was collected by repetitive vortexing and centrifugation. TMR-doped silica nanoparticles were rinsed with ethanol and acetone several times, and dried under vacuum at $100^{\circ} \mathrm{C}$ for $12 \mathrm{hr}$. Subsequently, silica nanoparticles were linked with PEG through two consecutive steps: i) the reaction between silanol groups on silica surfaces and aldehyde groups from 3-(trimethoxysilyl) propyl aldehyde (TPA) and ii) subsequent conjugation of PEG- $\mathrm{NH}_{2}$ (M.W. 3000) via a reactive schiff's base linkage. Particle morphology and size was observed by scanning electron microscope and transmission electron microscope (JEOL 2000FX). The presence of PEG attached on the silica surface was verified using Fourier Transform-Infrared Reflection Absorption Spectroscopy (FT-IRRAS) (data not shown).

\section{Isolation of adult guinea pig spinal cords and inducement of injury}

All guinea pigs used in this study were handled in accordance with Purdue Animal Care and Use Committee (PACUC) guidelines. Fifteen adult female guinea pigs, of the Hartley strain (300 plus grams) were anesthetized with an intramuscular injection of ketamine $(60 \mathrm{mg} / \mathrm{kg})$ and xylazine $(10 \mathrm{mg} / \mathrm{kg})$. Then they were perfused through the heart with approximately $500 \mathrm{ml}$ of sterile lactated ringer (SLR) solution to remove the blood. The vertebral column was then rapidly removed and a complete dorsal laminectomy performed along the length of the vertebral column, exposing the spinal cord. The spinal cord was carefully removed from vertebrae and immersed in cold, oxygenated Kreb's solution (124 mM NaCl, $2 \mathrm{mM} \mathrm{KCl}, 1.24 \mathrm{mM} \mathrm{KH_{2 }} \mathrm{PO}_{4}, 1.3 \mathrm{mM}$ $\mathrm{MgSO}_{4}, 1.2 \mathrm{mM} \mathrm{CaCl}, 10 \mathrm{mM}$ glucose, $26 \mathrm{mM}$ $\mathrm{NaHCO}_{3}$, and $10 \mathrm{mM}$ ascorbic acid).

A standardized compression injury was induced using a modified forceps possessing a detente by constant mechanical force for $15 \mathrm{sec}$ (Figure 1). Previously 
reported investigations of compound action potential (CAP) conduction through whole guinea pig spinal cord using the Double Sucrose Gap Isolation / Recording chamber (DSGR) verified the immediate and complete loss of conduction through such constant displacement compression injury produced in this way $[56,57]$.

\section{Evaluation of targeting ability of TMR-PSiNPs in the spinal cord tissues}

As nanoparticle targeting to a desired location improves therapeutic response, TMR-PSiNPs were applied to the segments of ex vivo spinal cord tissues containing compression injury and three different intact sites to evaluate the distribution and localization of the particles. The lesion was centrally located along the long axis of the cord, and the three uninjured sites of study were situated $1 \mathrm{~cm}, 2 \mathrm{~cm}$, and $3 \mathrm{~cm}$ from it (Figure 1). The compression injured spinal cord tissue was incubated in the suspension of TMR-PSiNPs for $15 \mathrm{~min}$ followed by the immersion in modified Krebs's solution for another $30 \mathrm{~min}$. The spinal cords were immediately fixed in $4 \%$ paraformaldehyde, sectioned with a freezing microtome with $50 \mu \mathrm{m}$ thick and evaluated with a fluorescent microscope using excitation/barrier wavelengths of 545/ $590 \mathrm{~nm}$, respectively. Labeling with a TMR dye was quantified using $\mathrm{NIH}$ Image $^{\mathrm{Ta}}$ software by statistically measuring fluorescence (minus background) intensity. As in prior studies the existence of differing levels of background fluorescence in the samples was normalized by measurement of fluorescence within standardized regions of background at a standard distance from the region of interest - and subtracting this to normalize this data; $[37,51]$.

For Scanning Electron Microscope (SEM) measurements, tissue sections containing particles were fixed in $2.5 \%$ glutaraldehyde, and dehydrated with increasing concentrations of ethanol by conventional methods. After drying, the silica nanoparticles were observed after a JEOL JFC-110 ion sputtering (Tokyo, Japan) and analyzed in a JEOL JSM-840 SEM. As another complementary test, the existence of particles within the spinal cord tissues was evaluated using Kratos Axis ULTRA X-ray Photoelectron Spectrometer.

\section{Statistical analysis}

Unless otherwise specified, unpaired student's $t$ test (for comparison of 2 groups) or one-way ANOVA and Post Hoc Newman Keul's test (for more than 2 groups) were used for statistical analyses (InStat ${ }^{\mathrm{Tm}}$ software). Normality was tested by Shapiro-Wilk test (STATA). Equal variances were tested by the method of Barlett for $n \geq 5$ (InStat ${ }^{\text {tw }}$ software), and by less than 2 -fold difference in $\mathrm{SD}$ for $\mathrm{n}<5$. Results are expressed as mean $\pm \mathrm{SD}$. $\mathrm{P}<0.05$ was considered statistically significant.

\section{Competing interests}

The authors declare they have no competing interests. There is no conflict of interest of any sort in the reporting of these data relative to any author.

\section{Authors' contributions}

YC drafted the manuscript and designed the experiments. $M Z$ and $B C$ performed the experiments. RBB is the Principle Investigator and Director of the CPR and is responsible for all elements of the research. All authors read and approved final manuscript.

\section{Acknowledgments}

We would gratefully like to thank Debra Bohnert for her expert surgery and handling of these whole animal experiments. We appreciate the excellent illustrations and graphics by Michel Schweinsberg, and the administrative assistance of Jennifer Danaher for manuscript preparation. This research was supported by General Funds from the CPR (State of Indiana HB 1440), and an endowment from Mrs. Mari Hulman George.

\section{Author details}

${ }^{1}$ Center for Paralysis Research, Department of Basic Medical Sciences, School of Veterinary Medicine, Purdue University, West Lafayette, IN 47907, USA. ${ }^{2}$ Weldon School of Biomedical Engineering, Purdue University, West Lafayette, IN 47907, USA. ${ }^{3}$ Molecular Imaging and Therapy Branch, National Cancer Center, 323 IIsan-ro, Goyang-si, Gyeonggi-do 410-769, Korea.

Received: 19 October 2011 Accepted: 6 September 2012

Published: 14 September 2012

\section{References}

1. McNally HA, Borgens RB: Three-Dimensional Imaging of Living and Dying Neurons with Atomic Force Microscopy. J Neurocyto/ 2004, 33:251-258.

2. Schwab JM, Leppert CA, Kaps K, Monnier PP: Functional recovery after spinal cord injury: basic science meets clinic. Trends Neurosci 2001, 24:437-439.

3. Schwab M: Repairing the Injured Spinal Cord. Scienc 2002, 295:1029-1031.

4. Borgens RB: Cellular engineering: molecular repair of membranes to rescue cells of the damaged nervous system. Neurosurgery 2001, 49:370-378. discussion 378-379.

5. Borgens RB, Shi R: Immediate recovery from spinal cord injury through molecular repair of nerve membranes with polyethylene glycol. FASEB J 2000, 14:27-35.

6. Bradbury E, McMahon S: Spinal cord repair strategies: why do they work? Nature Rev Neurosci 2006, 7:644-653.

7. Keirstead HS, Nistor G, Bernal G, Totoiu M, Cloutier F, Sharp K, Steward O: Human Embryonic Stem Cell-Derived Oligodendrocyte Progenitor Cell Transplants Remyelinate and Restore Locomotion after Spinal Cord Injury J Neurosci 2005, 29:4694-4705.

8. Coutts M, Keirstead HS: Stem cells for the treatment of spinal cord injury. Exp Neurol 2008, 209:368-377.

9. Borgens R, Shi R, Bohnert D: Behavioral Recovery From Spinal Cord Injury Following Delayed Application of Polyethylene Glycol. J Exp Biology 2002, 205:1-12.

10. Luo J, Borgens R, Shi R: Polyethylene glycol improves function and reduces oxidative stress in synaptosomes following spinal cord injury. J Neurotrauma 2004, 21:994-1007.

11. Serbest G, Horwitz J, Jost M, Barbee K: Mechanisms of cell death and neuroprotection by poloxamer 188 after mechanical trauma. FASEB $J$ 2006, 20:308-310.

12. Cadichon SB, Le H, Wright D, Curry D, Kang U, Frim DM: Neuroprotective effect of the surfactant poloxamer 188 in a model of intracranial hemorrhage in rats. J Neurosurg 2007, 106:36-40.

13. Koob AO, Borgens RB: Polyethylene glycol treatment after traumatic brain injury reduces beta-amyloid precursor protein accumulation in degenerating axons. J Neurosci Res 2006, 83:1558-1563.

14. Koob AO, Duerstock BS, Babbs CF, Sun Y, Borgens RB: Intravenous polyethylene glycol inhibits the loss of cerebral cells after brain injury. J Neurotrauma 2005, 22:1092-1111.

15. Laverty PH, Leskovar A, Breur GJ, Coates JR, Bergman RL, Widmer WR, Toombs JP, Shapiro S, Borgens RB: A preliminary study of intravenous surfactants in paraplegic dogs: polymer therapy in canine clinical $\mathrm{SCl}$. J Neurotrauma 2004, 21:1767-1777. 
16. Lee RC, River LP, Pan F, Wollman RS: Surfactant-induced sealing of electrpermeabilized skeletal muscle membranes in vitro. Proc Natl Acad Sci USA 1992, 89:4524-4528.

17. Merchant FA, Holmes WH, Capelli-schellpfeffer BS, Lee RC, Toner M: Poloxamer 188 enhances functional recovery of lethally heat-shocked fibroblasts. J Surgical Research 1998, 74:131-140.

18. Palmer JS, Cromie WJ, Lee RC: Surfactant administration reduces testicular ischemia-reperfusion injury. J Urol 1998, 159:2136-2139.

19. Borgens RB, Bohnert D, Duerstock B, Spomar D, Lee RC: Subcutaneous triblock copolymer produces recovery from spinal cord injury. J Neurosci Res 2004, 76:141-154.

20. Quinlan JG, Wong BL, Niemeier RT, McCullough AS, Levin L, Emanuele M: Poloxamer 188 failed to prevent exercise-induced membrane breakdown in mdx skeletal muscle fibers. Neuromuscul Disord 2006, 16:855-864.

21. Shi Y, Kim S, Huff TB, Borgens RB, Park K, Shi R, Cheng JX: Effective repair of traumatically injured spinal cord by nanoscale block copolymer micelles. Nat Nanotechnol 2009, 5:80-87.

22. Borgens RB, Bohnert D: Rapid recovery from spinal cord injury following subcutaneously administered polyethylene glycol. J Neurosci Res 2001, 66:1179-1186.

23. Donaldson J, Shi R, Borgens R: Polyethylene glycol rapidly restores physiological functions in damaged sciatic nerves of guinea pigs. Neurosurgery 2002, 50:147-156.

24. Koob AO, Colby JM, Borgens RB: Behavioral recovery from traumatic brain injury after membrane reconstruction using polyethylene glycol. J Biol Eng 2008, 2:9.

25. Ditor DS, John SM, Roy J, Marx JC, Kittmer C, Weaver LC: Effects of polyethylene glycol and magnesium sulfate administration on clinically relevant neurological outcomes after spinal cord injury in the rat. J Neurosci Res 2007, 85:1458-1467.

26. Detlof MR, Lavik E, Fisher LC, Langer R, Basso DM: Polyethylene Glycol Administration After Moderate Spinal Cord Injury Decreases Lesion Size and Improves Locomotor Recover. J Neurol Phys Ther 2006, 30:201. 210.1097/1001.NPT.0000281275.0000295819.cd

27. Detloff MR, Lavik E, Fisher LC, Langer R, Basso DM: Polyethylene glycol administration after moderate spinal cord injury decreases lesionsize and improves locomotor recovery. J Neurotrauma 2005, 22:1219.

28. Schneiderman S, Farber $J$, Baserga R: A simple method for decreasing the toxicity of polyethylene glycol in mammalian cell hybridization. Somat Cell Mol Genet 1979, 5:263-269.

29. Szabó G, Kiss A, Trón L: Permeabilization of lymphocytes with polyethylene glycol 1000. Discrimination of permeabilized cells by flow cytometry. Cytometry 1982, 3:59-63.

30. Cole A, Shi R: Prolonged focal application of polyethylene glycol induces conduction block in guinea pig spinal cord white matter. Toxicol Vitr 2005, 19:215-220.

31. Webster R, Elliott V, Park BK, Walker D, Hankin M, Taupin P: PEG and PEG conjugates toxicity: towards an understanding of the toxicity of PEG and its relevance to PEGylated biologicals. In PEGylated Protein Drugs: Basic Science and Clinical Application. Edited by Veronese FM. Switzerland: Birkhäuser Basel; 2009:127-146. Milestones in Drug Therapy.

32. Brent J: Current management of ethylene glycol poisoning. Drugs 2001, 61:979-988.

33. Caravati EM, Erdman AR, Christianson G, Manoguerra AS, Booze LL, Woolf $A D$, Olson KR, Chyka PA, Scharman EJ, Wax PM, et al: Ethylene glycol exposure: an evidence-based consensus guideline for out-of-hospital management. Clin Toxicol (Phila) 2005, 43:327-345.

34. Cho Y, Shi R, Borgens R, Ivanisevic A: Repairing the Damaged Spinal Cord and Brain with Nanomedicine. Small 2008, 4:1676-1681.

35. Cho Y, Shi R, Borgens RB: Chitosan nanoparticle-based neuronal membrane sealing and neuroprotection following acrolein-induced cell injury. J Bio Eng 2010, 29:2

36. Cho Y, Shi R, Ivanisevic A, Borgens RB: The Functionalized Mesoporous Silica Nanoparticles (MSNs) Based Drug Delivery System to Rescue Acrolein-Mediated Cell Death. Nanomedicine 2008, 3:507-519.

37. Cho Y, Shi R, Ivanisevic A, Borgens RB: Functional Silica NanoparticleMediated Neuronal Membrane Sealing Following Traumatic Spinal Cord Injury. J Neurosci Res 2010, 88:1433-1444.

38. Mohanraj VJ, Chen Y: Nanoparticles - A Review. Trop J Pharm Res 2006, 5:561-573.
39. Liu-Snyder $P$, Logan M, Shi R, Smith D, Borgens R: Neuroprotection from secondary injury by polyethylene glycol requires its internalization. $J$ Exp Biol 2007, 210:1-8

40. Liu-Snyder P, Logan MP, Shi R, Smith DT, Borgens RB: Neuroprotection from secondary injury by polyethylene glycol requires its internalization. J Exp Biol 2007, 210:1455-1462.

41. Cho Y, Borgens RB: Polymer and nano-technology applications for repair and reconstruction of the central nervous system. Exp Neurol 2012 , 233:126-144.

42. Borgens RB, Liu-Snyder P: Understanding Secondary Injury. The Quarterly Review Of Biology 2012, 87:89-127.

43. Bittner GD, Ballinger ML, Raymond MA: Reconnection of severed nerve axons with polyethylene glycol. Brain Res 1986, 367:351-355.

44. Lee RC: Cytoprotection by Stabilization of Cell Membranes. Annals New Your Academy of Sciences 2002, 961:271-275.

45. Lentz B: PEG as a tool to gain insight into membrane fusion. Eur Biophys $J$ 2007, 36:315-326.

46. MacDonald Rl: Membrane fusion due to dehydration by polyethylene glycol, dextran, or sucrose. Biochemistry 1985, 24:4058-4066.

47. Weinreb G, Lentz BR: Analysis of membrane fusion as a two-state sequential process: evaluation of the stalk model. Biophys J 2007, 92:4012-4029.

48. Borgens RB, Jaffe LF, Cohen MJ: Large and persistent electrical currents enter the transected lamprey spinal cord. Proc Natl Acad Sci U S A 1980, 77:1209-1213

49. Borgens RB: Artificially controlling axonal regeneration and development by applied electric fields, Electric Fields in Vertebrate Repair. New York: Alan R. Liss; 1989:117-170.

50. Zuberi M, Liu-Snyder P, UI Haque A, Porterfield DM, Borgens RB: Large naturally-produced electric currents and voltage traverse damaged mammalian spinal cord. J Biol Eng 2008, 2:17.

51. Cho $Y$, Shi R, Borgens RB: Chitosan produces potent neuroprotection and physiological recovery following traumatic spinal cord injury. J Exp Biol 2010, 213:1513-1520.

52. Fang $N$, Chan V: Chitosan-induced restructuration of a mica-supported phospholipid bilayer: an atomic force microscopy study. Biomacromolecules 2003, 4:1596-1604.

53. Fang $\mathrm{N}$, Chan V, Mao HQ, Leong KW: Interactions of phospholipid bilayer with chitosan: effect of molecular weight and $\mathrm{pH}$. Biomacromolecules 2001, 2:1161-1168

54. Koob A, Duerstock B, Babbs C, Sun Y, Borgens R: Intravenous polyethylene glycol inhibits the loss of cerebral cells after brain injury. J Neurotrauma 2005, 22:1092-1111.

55. Koob A, Borgens R: Polyethylene Glycol Treatment After Traumatic Brain Injury Reduces Amyloid Precursor Protein Accumulation in Degenerating Axons. J Neurosci Res 2006, 83:1558-1563.

56. Shi R, Borgens RB: Anatomical repair of nerve membranes in crushed mammalian spinal cord with polyethylene glycol. J Neurocytol 2000, 29:633-643.

57. Luo J, Borgens RB, Shi R: Polyethylene glycol immediately repairs neuronal membranes and inhibits free radical production after acute spinal cord injury. J Neurochemistry 2002, 83:471-480.

doi:10.1186/1754-1611-6-18

Cite this article as: Chen et al: Affinity for, and localization of, PEGfunctionalized silica nanoparticles to sites of damage in an ex vivo spinal cord injury model. Journal of Biological Engineering 2012 6:18. 